\title{
Apoio a Infraestrutura Robótica Baseada em Arcabouço ROS para um Miniveículo Elétrico
}

\section{Guilherme L. Paiva*, Niederauer Mastelari}

\section{Resumo}

Dado o contexto "todo-terreno" do miniveículo VERDE, é fundamental a robustez dos sistemas de controle e sensoriamento a variações bruscas de relevo e ao deslizamento das rodas. Neste contexto, a odometria tradicional através dos encoders dos motores pode apresentar um grande erro cumulativo, ao passo de que o sistema de GPS também não possui precisão suficiente para uma boa localização do veículo. Este trabalho tratará da escolha e do estudo de viabilidade da implementação do sistema de percepção sensorial do Veículo Elétrico Robótico com Diferencial Eletrônico (VERDE) através de visão computacional, buscando o aproveitamento das características complementares das câmeras para solucionar os problemas descritos, adotando como arcabouço de software o sistema Robot Operating System (ROS).

\section{Palavras-chave:}

Robótica Móvel, Veículos Autônomos, Robot Operating System

\section{Introdução}

Neste trabalho serão tratados os estudos desenvolvidos no primeiro semestre de atividades de iniciação científica, correspondente ao estudo da infraestrutura já implantada, o uso do ambiente de simulações Gazebo e a revisão da literatura de visão computacional necessários para a implementação dos sistemas

\section{Resultados e Discussão}

O veículo elétrico miniaturizado utilizado como plataforma para esse trabalho é um veículo Baja $5 \mathrm{~b} 2.0$ modificado, equipado com uma unidade de medidas inerciais X-Sens, um sensor laser Hokuyo, uma câmera Pointgrey, três motores maxon, sendo dois para as rodas traseiras e um para o mecanismo de direção, um computador Intense com processador Intel Core i7 e baterias de polímero de lítio para a alimentação. (A. Ribeiro et al, L. Nogueira et al)

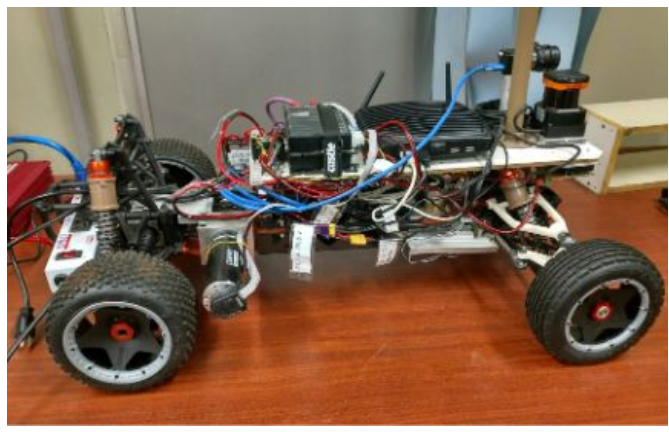

Figura 1. Miniveículo elétrico VERDE.

O uso do arcabouço ROS e sua estrutura de comunicação entre nós permite $\mathrm{o}$ aproveitamento de diversos algorítmos já implementados, como drivers de sensores, estimadores e controladores.

A implementação do sistema de odometria visual e mapeamento se dará com a adaptação de algorítmos já implementados de detecção e correspondência de características como o ORB, que possui licença opensource e apresentou uma redução do tempo de execução e memória utilizada de mais de $90 \%$ em relação a algorítmos mais antigos como o SURF (Tareen et al, E. Rublee et al).

\section{Conclusões}

O trabalho desenvolvido até o momento mostra a viabilidade da implementação do sistema de visão computacional no veículo, tanto na odometria visual como para o mapeamento do ambiente. É esperado que ao fim do período de atividades seja realizada a implementação do sistema visual inercial fracamente acoplado, proposto em (P. Corke et al) com ganhos de desempenho do veículo em ambiente off road.

\section{Agradecimentos}

Gostaria de agradecer aos meus orientadores Niederauer Mastelari e André Ricardo Fioravanti, coorientador Mauro Koyama e aos alunos dos laboratórios ACCES e LEIA da FEM-UNICAMP por todo o apoio oferecido no andamento deste trabalho.

Agradeço também ao $\mathrm{PIBIC/CNPq}$ pelo financiamento deste projeto de iniciação científica e à FAPESP pelo financiamento do projeto VERDE e Auto_VERDE, sem os quais este trabalho não seria possível.

A. Ribeiro, L. G. Bizarro Mirisola, M. F Koyama, E. Paiva, S. Bueno, and L. Nogueira, "Unisensory intra-row navigation strategy for orchards environments based on sensor laser," 012018.

L. Nogueira, M. F. Koyama, R. De Angelis Cordeiro, A. Ribeiro, S. Bueno, and E. Paiva,"A miniaturized four-wheel robotic vehicle for autonomous driving research in off-road scenarios," 012018

Tareen, Shaharyar Ahmed Khan \& Saleem, Zahra. (2018). A comparative analysis of SIFT, SURF, KAZE, AKAZE, ORB, and BRISK. 10.1109/ICOMET.2018.8346440.

P. Corke, J. Lobo, and J. Dias, "An introduction to inertial and visual sensing," I. J. Robotic Res., vol. 26, pp. 519-535, 062007.

E. Rublee, V. Rabaud, K. Konolige, and G. Bradski, "Orb: An efficient alternative to sift or surf," inProceedings of the 2011 International Conference on Computer Vision , ICCV'11, (Washington, DC, USA), pp. 2564-2571, IEEE Computer Society, 2011 